\title{
The effect of stellar compressibility and non-resonant dynamic tides on the apsidal-motion rate in close binaries
}

\author{
B. Willems ${ }^{1}$ and A. Claret $^{2}$ \\ 1 Department of Physics and Astronomy, The Open University, Walton Hall, Milton Keynes, MK7 6AA, UK \\ e-mail: B.Willems@open.ac.uk \\ 2 Instituto de Astrofísica de Andalucía, CSIC, Apartado 3004, 18080 Granada, Spain \\ e-mail: claret@iaa.es
}

Received 26 September 2002 / Accepted 18 November 2002

\begin{abstract}
The paper is devoted to the analysis of the systematic deviations between the classical formula for the rate of secular apsidal motion in close binaries and the formula established within the framework of the theory of dynamic tides. The basic behaviour is found to be governed by the dependency of the forcing frequencies on the orbital period and the rotational angular velocity, irrespective of the stellar model. For binaries with short orbital periods a good agreement between the classical apsidalmotion formula and the formula established within the framework of the theory of dynamic tides is only possible for certain values of the rotational angular velocity.
\end{abstract}

Key words. binaries: close - stars: oscillations - celestial mechanics

\section{Introduction}

In close binary systems of stars, the perturbation of the external gravitational field due to the tidal and rotational distortions of the component stars yields a periodic shift of the position of the periastron. The effect was first studied by Russell (1928), and subsequently by Cowling (1938) and Sterne (1939). The latter author derived a formula for the rate of secular apsidal motion based on the assumption that the orbital period is long in comparison to the periods of the free oscillation modes of the component stars. The validity of this assumption has been investigated extensively by, e.g., Papaloizou \& Pringle (1980), Smeyers et al. (1991), Quataert et al. (1996), Smeyers et al. (1998), and Smeyers \& Willems (2001). Smeyers \& Willems (2001) found the classical apsidal-motion formula to be valid up to high orbital eccentricities as long as the orbital and rotational angular velocities remain small. For binaries with higher orbital and rotational angular velocities, the apsidal-motion rates predicted by the classical formula deviate from those predicted by the theory of dynamic tides due the effects of the compressibility of the stellar fluid and the possibility of resonances between dynamic tides and free oscillation modes of the component stars.

One of the most uncertain parameters in the analysis of the dynamics of a close binary is the rotational angular velocity of

Send offprint requests to: $\mathrm{B}$. Willems,

e-mail: B.Willems@open.ac.uk the component stars. Claret \& Willems (2002) therefore considered the effects of dynamic tides on the apsidal-motion rate as a function of the rotational angular velocity for a carefully selected sample of eclipsing binaries with accurate determinations of the stellar masses and radii and the apsidal-motion rate. The authors found that even for binaries with short orbital periods, a good agreement between the classical apsidal-motion formula and the formula established within the framework of the theory of dynamic tides is possible for certain values of the rotational angular velocity.

In this research note, we aim to shed more light on the results found by Claret \& Willems (2002) by examining more closely the role of the forcing frequencies and the effects of the compressibility of the stellar fluid on the rate of secular apsidal motion due to the tidal distortions of close binary components. In Sects. 2 and 3, we recall the basic assumptions and the equations governing the rate of secular change of the longitude of the periastron in the classical framework and in the framework of the theory of dynamic tides. In Sect. 4, we illustrate the behaviour of the relative deviations between the classical apsidal-motion formula and the formula established within the framework of the theory of dynamic tides as a function of the rotational angular velocity in the case of an evolved $5 M_{\odot}$ mainsequence star. In Sects. 5 and 6, we unravel this behaviour by looking at the effects of the forcing frequencies and the stellar model separately. In the final section, we briefly summarise our conclusions. 


\section{Basic assumptions}

Consider a close binary system of stars with masses $M_{1}$ and $M_{2}$ that are orbiting around each other under the influence of their mutual gravitational force. Let $P_{\text {orb }}$ be the orbital period, $a$ the semi-major axis, and $e$ the orbital eccentricity. We assume the first star to rotate uniformly around an axis perpendicular to the orbital plane with an angular velocity $\Omega$ whose magnitude is small in comparison to the stellar break-up speed $\Omega_{c}$. The companion star is treated as a point mass.

Furthermore, we denote by $\boldsymbol{r}=(r, \theta, \phi)$ a system of spherical coordinates with respect to an orthogonal frame of reference that is corotating with the star. The potential governing the tidal force exerted by the companion can then be expanded in terms of unnormalised spherical harmonics $Y_{\ell}^{m}(\theta, \phi)$ and in Fourier series in terms of multiples of the companion's mean motion $n$ as

$$
\begin{gathered}
\varepsilon_{T} W(\boldsymbol{r}, t)=-\varepsilon_{T} \frac{G M_{1}}{R_{1}} \sum_{\ell=2}^{4} \sum_{m=-\ell}^{\ell} \sum_{k=-\infty}^{\infty} \\
c_{\ell, m, k}\left(\frac{r}{R_{1}}\right)^{\ell} Y_{\ell}^{m}(\theta, \phi) \exp \left[\mathrm{i}\left(\sigma_{T} t-k n \tau\right)\right]
\end{gathered}
$$

(e.g. Polfliet \& Smeyers 1990; Smeyers et al. 1998). In this expansion, $G$ is the Newtonian constant of gravitation, $R_{1}$ is the equilibrium radius of the uniformly rotating star, $\varepsilon_{T}=$ $\left(R_{1} / a\right)^{3} M_{2} / M_{1}$ is a small dimensionless parameter, $\tau$ is a time of periastron passage, and $\sigma_{T}=k n+m \Omega$ is a forcing angular frequency with respect to the corotating frame of reference. For a definition of the Fourier coefficients $c_{\ell, m, k}$, we refer to Smeyers et al. (1998). They are equal to zero for odd values of $\ell+|m|$ and, for a given eccentricity, decrease rapidly with increasing values of $k$.

For the remainder of the paper, we restrict ourselves to the contributions of the dominant terms associated with $\ell=2$ in the expansion of the tide-generating potential.

\section{The apsidal-motion rate due to the tidal deformations of binary components}

The most commonly used formula for the rate of secular change of the longitude of the periastron due to the tidal deformations of the components of a close binary was derived by Cowling (1938) and Sterne (1939) on the assumption that the orbital period is long in comparison with the free harmonic periods of the component stars. The rate of secular apsidal motion is then given by

$$
\left(\frac{\mathrm{d} \varpi}{\mathrm{d} t}\right)_{\text {classical }}=\left(\frac{R_{1}}{a}\right)^{5} \frac{M_{2}}{M_{1}} \frac{2 \pi}{P_{\text {orb }}} k_{2} 15 f\left(e^{2}\right),
$$

where $\varpi$ is the longitude of the periastron, $k_{2}$ is the classical apsidal-motion constant, and

$f\left(e^{2}\right)=\left(1-e^{2}\right)^{-5}\left(1+\frac{3}{2} e^{2}+\frac{1}{8} e^{4}\right)$.
A formula for the rate of secular change of the longitude of the periastron taking into account the effects of dynamic tides was derived by Smeyers et al. (1998) by adding the contributions to the apsidal-motion rate stemming from the various terms in Expansion (1) of the tide-generating potential. The resulting formula takes the form

$$
\begin{gathered}
\left(\frac{\mathrm{d} \varpi}{\mathrm{d} t}\right)_{\mathrm{dyn}}=\left(\frac{R_{1}}{a}\right)^{5} \frac{M_{2}}{M_{1}} \frac{2 \pi}{P_{\mathrm{orb}}}\left[2 k_{2} G_{2,0,0}+4 \sum_{k=1}^{\infty}\right. \\
\left.\left(F_{2,0, k} G_{2,0, k}+F_{2,2, k} G_{2,2, k}+F_{2,-2, k} G_{2,-2, k}\right)\right],
\end{gathered}
$$

where the constants $F_{2, m, k}$ render the response of the star to the various forcing angular frequencies $\sigma_{T}$, and the coefficients $G_{2, m, k}$ are functions of the orbital eccentricity. For a definition of the constants $F_{2, m, k}$ and the coefficients $G_{2, m, k}$, we refer to Smeyers et al. (1998) and Smeyers \& Willems (2001). For a given eccentricity, the coefficients $G_{2, m, k}$ decrease rapidly with increasing values of $k$.

For binaries with short orbital periods or rapidly rotating component stars, the rates of secular apsidal motion predicted by the classical formula deviate from the corresponding rates predicted by the formula established in the framework of the theory of dynamic tides due to the increased role of the stellar compressibility at higher forcing frequencies and due to resonances of dynamic tides with free oscillation modes of the component stars (Smeyers \& Willems 2001). The extent of the deviations depends on the evolutionary stage of the star due to the increase of the stellar radius and the redistribution of the mass as the star evolves on the main sequence (Willems \& Claret 2002). In the present investigation, we focus on the role of the forcing frequencies and the compressibility of the stellar fluid. In analogy to previous investigations (Smeyers \& Willems 2001; Willems \& Claret 2002; Claret \& Willems 2002), we study the deviations between the apsidal-motion rates predicted by the classical formula and the rates predicted by the formula taking into account the effects of dynamic tides by means of the relative difference

$\Delta=\frac{(\mathrm{d} \varpi / \mathrm{d} t)_{\text {classical }}-(\mathrm{d} \varpi / \mathrm{d} t)_{\mathrm{dyn}}}{(\mathrm{d} \varpi / \mathrm{d} t)_{\mathrm{dyn}}}$.

\section{Relative deviations for a $5 M_{\odot}$ main-sequence star}

We illustrate the behaviour of the relative differences $\Delta$ as a function of the rotational angular velocity $\Omega$ in the case of a $5 M_{\odot}$ main-sequence star with a central hydrogen abundance $X_{\mathrm{c}}=0.36$ and a radius $R_{1}=3.8 R_{\odot}$. Since the rotational angular velocity $\Omega$ is assumed to be small, we neglect the effects of the Coriolis force and the centrifugal force for the determination of the constants $F_{2, m, k}$. The tides then correspond to those of a non-rotating spherically symmetric equilibrium star (for more details see, e.g., Willems \& Claret 2002).

The resulting relative deviations $\Delta$ are presented in Fig. 1 for the orbital eccentricity $e=0.4$ and for orbital periods ranging from 5.0 to 12.5 days. For convenience, the rotational angular velocity $\Omega$ is expressed in units of the orbital angular 


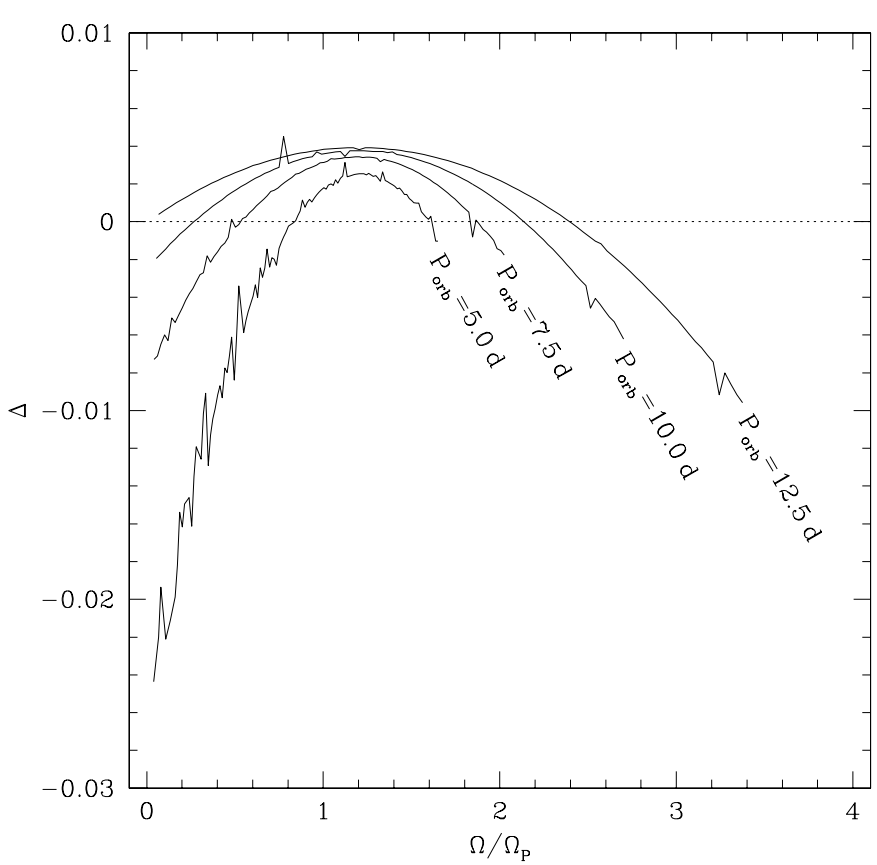

Fig. 1. The relative differences $\Delta$ for the evolved $5 M_{\odot}$ main-sequence model, the orbital eccentricity $e=0.4$, and the orbital periods $P_{\text {orb }}=$ 5.0, 7.5, 10.0, 12.5 days.

velocity $\Omega_{\mathrm{P}}$ at the periastron of the relative orbit. The peaks in the curves correspond to resonances between dynamic tides and free oscillation modes. Since we are here interested in the systematic trend caused by the effects of the forcing frequencies and the compressibility of the stellar fluid rather than in a detailed treatment of resonant dynamic tides, we did not attempt to find all possible resonances for each orbital configuration. For a detailed treatment of the resonances, we refer the reader to Smeyers \& Willems (2001) and Willems \& Claret (2002).

In the limiting case where $\Omega \rightarrow 0$, the forcing frequencies $\sigma_{T}$ are determined solely by the mean motion $n$. As shown by Smeyers \& Willems (2001), the relative differences $\Delta$ are then generally negative and larger in absolute value for shorter orbital periods. For larger values of $\Omega$, the relative differences $\Delta$ increase, reach a positive maximum, and subsequently decrease to become negative. Hence, we find two values of the rotational angular velocity for which the relative differences $\Delta$ become zero. These values depend on both the period and the eccentricity of the orbit, and do not necessarily correspond to synchronised rotation with the orbital angular velocity at the periastron of the relative orbit. We will further scrutinise this behaviour in the following section.

\section{The role of the forcing frequencies}

Due to the expansion of the tide-generating potential in Fourier series in terms of multiples of the companion's mean motion, the tidally distorted star is subjected to an infinite number of forcing angular frequencies $\sigma_{T}$ (see Eq. (1)). For a given orbital eccentricity, the largest differences between the classical apsidal-motion formula and the formula established within the framework of the theory of dynamic tides can be expected to occur when high forcing frequencies are associated with large Fourier coefficients $c_{2, m, k}$. Vice versa, the smallest differences can be expected to occur when the dominant terms in the expansion of the tide-generating potential all have small forcing frequencies.

A similar reasoning applies to Expansion (4) which renders the apsidal-motion rate within the framework of the theory of dynamic tides. Since the system of differential equations governing non-resonant dynamic tides in components of close binaries depends on the square of the forcing frequency, the deviations of the constants $F_{2, m, k}$ from the classical apsidal-motion constant $k_{2}$ are of the order of $\sigma_{T}^{2}$. Because of this, Smeyers \& Willems (2001) and Claret \& Willems (2002) were able to approximate the systematic deviations between the classical apsidal-motion formula and the formula established within the framework of the theory of dynamic tides by second-degree polynomials in the companion's mean motion $n$ or the star's rotational angular velocity $\Omega$. In the present context, this property implies that the differences between the two formulae can be expected to be large when high forcing frequencies are associated with large coefficients $G_{2, m, k}$.

In order to shed some more light on this, we examine the behaviour of the function

$S_{1}\left(P_{\text {orb }}, \Omega\right)=\sum_{m=-2}^{2} \sum_{k=0}^{\infty} \sigma_{T}^{2} G_{2, m, k}^{2}$.

The function mimics the behaviour described above in the sense that it will be large when high forcing frequencies are associated with large coefficients $G_{2, m, k}$.

The variations of $S_{1}$ as a function of the rotational angular velocity $\Omega$ are displayed in Fig. 2 in the case of the orbital eccentricity $e=0.4$ and orbital periods ranging from 5.0 to 12.5 days. For given values of $e$ and $P_{\text {orb }}$, the function $S_{1}$ initially decreases with increasing values of $\Omega$. The decrease is caused by the decrease of the forcing frequencies associated with $m=-2$, which generally provide the dominant contributions to $S_{1}$. As $\Omega$ becomes larger, $S_{1}$ reaches a minimum when the forcing frequencies associated with the largest coefficients $G_{2,-2, k}$ are close to zero. The frequencies associated with $m=-2$ subsequently become more and more negative, resulting in an indefinite increase of the function $S_{1}$.

As a preliminary conclusion we can thus say that the opposite of the function $S_{1}$ shows the same qualitative behaviour as the relative differences $\Delta$ shown in Fig. 1. In the next section, we will make this even clearer by adding some information on the stellar model to the definition of $S_{1}$.

\section{The role of the stellar model}

The response of a particular stellar model to the tidal forcing of a close companion is determined by the constants $F_{2, m, k}$. For low-frequency, non-resonant dynamic tides in stars with a radiative envelope, these constants can be approximated by their asymptotic representation

$F_{2, m, k}^{(\mathrm{a})}=k_{2}-\frac{R_{1}^{3}}{G M_{1}} \frac{\sigma_{T}^{2}}{12}\left[\left(\frac{\mathrm{d} \xi_{2}}{\mathrm{~d} r}\right)_{R_{1}}+2 \frac{\xi_{2}\left(R_{1}\right)}{R_{1}}\right]$ 


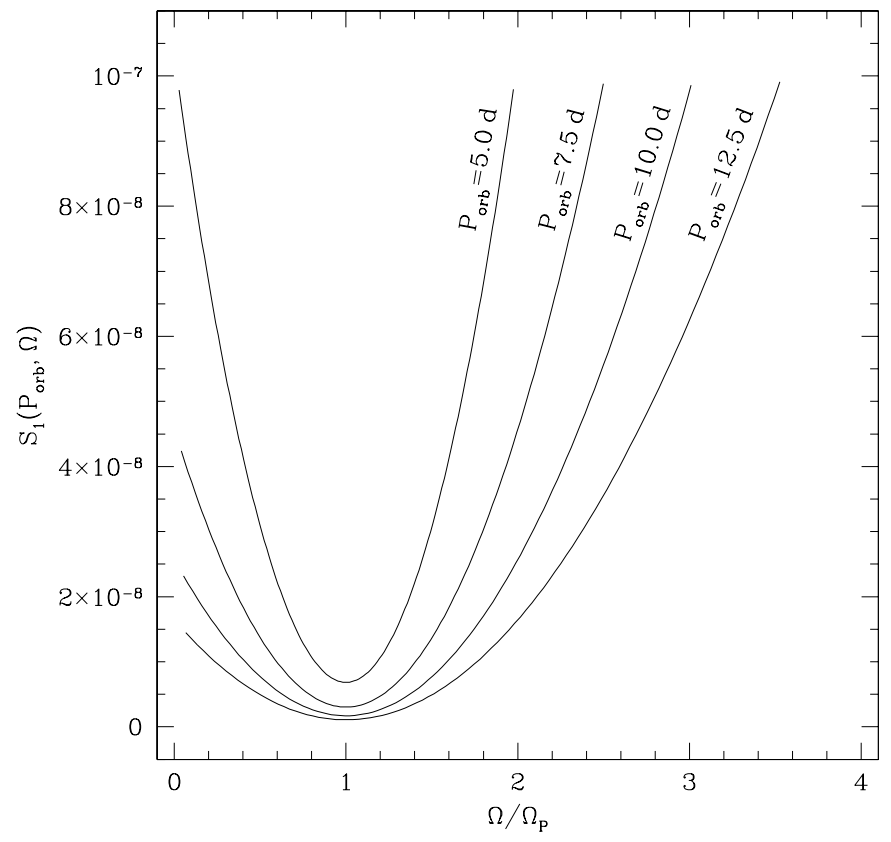

Fig. 2. The variation of $S_{1}\left(P_{\mathrm{orb}}, \Omega\right)$ as a function of the rotational angular velocity $\Omega$ for the same orbital eccentricity and orbital periods as considered in Fig. 1.

where $\xi_{2}(r)$ is a solution of the homogeneous second-order differential equation

$\frac{\mathrm{d}^{2} \xi_{2}}{\mathrm{~d} r^{2}}+2\left[\frac{1}{m(r)} \frac{\mathrm{d} m(r)}{\mathrm{d} r}-\frac{1}{r}\right] \frac{\mathrm{d} \xi_{2}}{\mathrm{~d} r}-\frac{4}{r^{2}} \xi_{2}=0$

(Smeyers et al. 1998). In the latter equation, $m(r)$ is the mass contained inside the sphere with radius $r$. The solution of Eq. (8) must furthermore remain finite at $r=0$ and satisfy the boundary condition

$\left(\frac{\mathrm{d} \xi_{2}}{\mathrm{~d} r}\right)_{R_{1}}+\frac{\xi_{2}\left(R_{1}\right)}{R_{1}}=5$.

The asymptotic approximation for the constants $F_{2, m, k}$ is valid for forcing frequencies $\sigma_{T} \ll\left(G M_{1} / R_{1}^{3}\right)^{1 / 2}$. Higher forcing frequencies, such as those associated with large values of $k$, require additional terms in the asymptotic approximation. However, the second-order approximation given by Eq. (7) is sufficient to further clarify the qualitative behaviour of the relative differences $\Delta$ observed in Fig. 1.

We proceed in a similar way as for the introduction of the function $S_{1}$ in the previous section, but now take into account the structure of the stellar model by defining a function $S_{2}$ as

$S_{2}\left(P_{\mathrm{orb}}, \Omega\right)=\sum_{m=-2}^{2} \sum_{k=0}^{\infty} F_{2, m, k}^{(\mathrm{a})} G_{2, m, k}^{2}$.

The function can be rewritten in terms of $S_{1}$ as

$$
\begin{aligned}
& S_{2}\left(P_{\text {orb }}, \Omega\right)=k_{2} \sum_{m=-2}^{2} \sum_{k=0}^{\infty} G_{2, m, k}^{2} \\
& -\frac{1}{12} \frac{R_{1}^{3}}{G M_{1}}\left[\left(\frac{\mathrm{d} \xi_{2}}{\mathrm{~d} r}\right)_{R_{1}}+2 \frac{\xi_{2}\left(R_{1}\right)}{R_{1}}\right] S_{1}\left(P_{\mathrm{orb}}, \Omega\right) .
\end{aligned}
$$

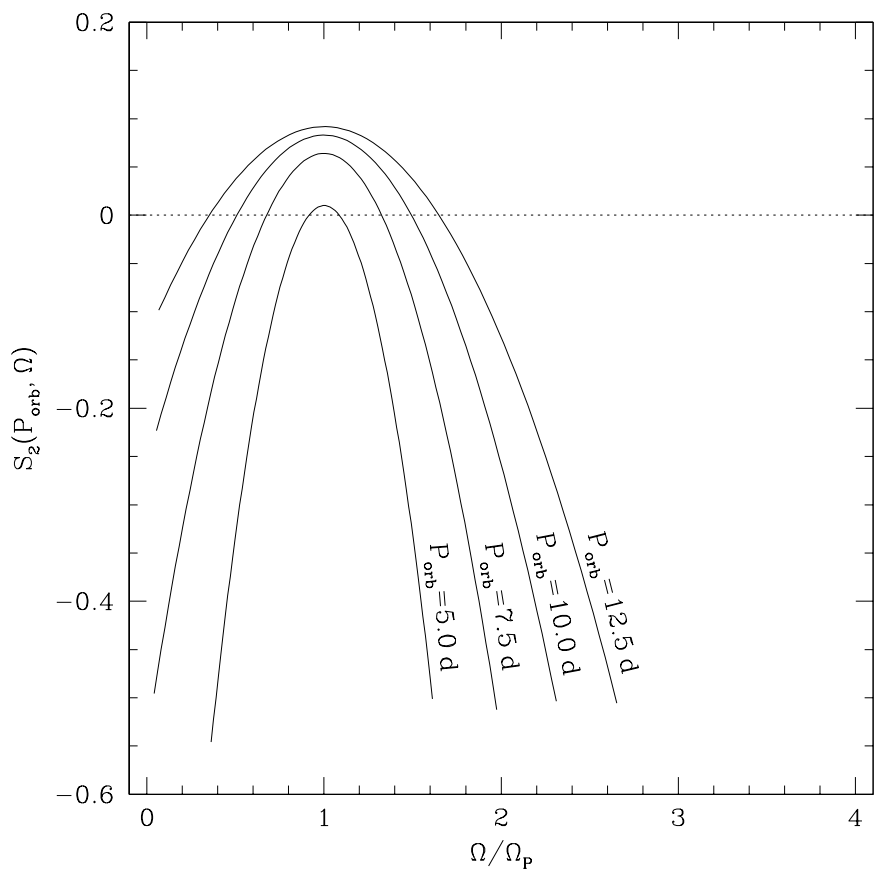

Fig. 3. The variation of $S_{2}\left(P_{\text {orb }}, \Omega\right)$ for the evolved $5 M_{\odot}$ mainsequence model and the same orbital eccentricity and orbital periods as considered in Fig. 1.

For a given stellar model and a given orbital eccentricity, the first term is then seen to be constant, while the second term depends on the orbital period and the rotational angular velocity through the function $S_{1}$.

The variations of $S_{2}$ as a function of the rotational angular velocity $\Omega$ are displayed in Fig. 3 for the same $5 M_{\odot}$ mainsequence model used in Sect. 4. The qualitative behaviour of $S_{2}$ is essentially the same as that of $-S_{1}$. In addition, the inclusion of the model dependent factors introduces two zeros in the curves, similar to the behaviour of the relative differences $\Delta$ displayed in Fig. 1.

\section{Summary}

In this research note, we scrutinised the behaviour of the systematic relative deviations between the classical formula for the rate of secular apsidal motion and the formula established within the framework of the theory of dynamic tides as a function of the often uncertain rotational angular velocity of the component stars. We found that when the effects of resonant dynamic tides are neglected, the basic behaviour of the relative differences is governed by the dependency of the forcing frequencies on the orbital period and the rotational angular velocity, irrespective of the stellar model. For a given value of the orbital eccentricity, a good agreement between the classical apsidal-motion formula and the formula established within the framework of the theory of dynamic tides is possible even for binaries with short orbital periods, but only for certain values of the rotational angular velocity. 
Acknowledgements. BW and AC acknowledge the support received by the British Particle Physics and Astronomy Research Council (PPA/G/S/1999/00127) and the Spanish DGI (AYA2000-2559), respectively.

\section{References}

Claret, A., \& Willems, B. 2002, A\&A, 388, 518

Cowling, T. G. 1938, MNRAS, 98, 734

Papaloizou, J., \& Pringle, J. E. 1980, MNRAS, 193, 603
Polfliet, R., \& Smeyers, P. 1990, A\&A, 237, 110

Quataert, E. J., Kumar, P., \& Ao, C. O. 1996, ApJ, 463, 284

Russell, H. N. 1928, MNRAS, 88, 641

Smeyers, P., Van Hout, M., Ruymaekers, E., \& Polfliet, R. 1991, A\&A, 248, 94

Smeyers, P., \& Willems, B. 2001, A\&A, 373, 173

Smeyers, P., Willems, B., \& van Hoolst, T. 1998, A\&A, 335, 622

Sterne, T. E. 1939, MNRAS, 99, 451

Willems, B., \& Claret, A. 2002, A\&A, 382, 1009 